\title{
MorphAn: A new software tool to assess sandy coasts
}

\author{
Q.J. Lodder ${ }^{1}$ and P.F.C. van Geer ${ }^{2}$ \\ ${ }^{1}$ Rijkswaterstaat, waterdienst, P.O.Box 17, 8224 AD, Lelystad, the Netherlands \\ ${ }^{2}$ Deltares, P.O.Box 177, 2600 MH, Delft, the Netherlands, Pieter.vanGeer@deltares.nl
}

\begin{abstract}
We present a new software tool called "MorphAn" that enables easy assessment of sandy coasts. MorphAn provides the possibility to import data of various formats. The software pays special attention to coastal data provided in the so-called Jarkus format. Data can be analyzed with a GIS based map view. Simultaneously an overview of the profile development in time or a more detailed picture of the actual measurements is given. Furthermore, MorphAn includes the possibility to assess coasts for dune safety according the safety assessment rules VTV2006. The software program gives a graphically presentation of the calculation results of dune erosion according to the Duros + and $\mathrm{D}++$ model as well as calculation results of the normative erosion points. MorphAn also provides the possibility to calculate and visualize the momentary coastline (MKL) and the expected near future coast line position (TKL). The software is freely available and expected to become open source in 2012.
\end{abstract}

\section{INTRODUCTION}

This short paper will introduce you to a new and freely available software tool called "MorphAn". MorphAn enables the user to assess the safety and beach development of sandy coasts.

In 2008 the Dutch Ministry of Infrastructure and the Environment commissioned Deltares to start the build of a new software tool. The aim of this software is to support assessment of dune safety and beach development. Rijkswaterstaat plans to present MorphAn as its official and supported tool for assessment of dune safety in The Netherlands. From the start of the project, one of the main goals has been to release MorphAn as a freely available, preferably open source, software tool. At this moment, the beta version of MorphAn is freely available. Release of the source code is expected at the end of 2012.

Data analysis, Dune safety assessment and coastal development assessment are the three pillars that form the basis of MorphAn. This paper will discuss the figures and tools included in MorphAn to support each of these pillars.

\section{DATA ANALYSES}

One of the most important aspects of assessing dune safety or coastal development is analyzing the data that is used to calculate a dune crest retreat point after a storm or momentary coastline. MorphAn offers the ability to import and analyze so-called jarkus measurements (yearly measurements of coastal profiles). A default jarkus dataset that includes most yearly measurements of the Dutch coast between 1965 and 2011 accompanies the software. Next to that, there is also the possibility to import user defined datasets or expand the default dataset with additional measurements.

Once the data is imported, it is possible to specify a list of locations that is used in all operations throughout the program. MorphAn ignores all measurements at locations not in that list.
To accommodate a thorough analysis of the imported data MorphAn provides various types of views that enable the user to analyze the data at different levels of aggregation and along the various dimensions in time and space. This section will introduce you briefly to the types of figures included in MorphAn.

\section{Map overview}

The map overview provides a GIS based map that gives a top view of the measurements in space and time. Figure 1 gives an example of the jarkus measurements at Texel for 2011. Blue lines represent the wet parts of the measurements, whereas the sandy colored lines represent dry measurements. Interpolated profile parts can be recognized by the tomato red colored lines. MorphAn also includes a time navigator to control the period that is presented on the map.

Due to its GIS nature it is easy to expand the map by including for example shapefiles as is shown by including the Texel district boundaries and names in Figure 1.

\section{Transect side view}

The transect side view (Figure 2) shows a single jarkus measurement in the local coordinate system (RSP). The side view distinguishes wet measurements, dry measurements and the interpolated profile part with the same coloring as the map overview. Furthermore, the side view interrupts the plotted line whenever more than 10 meter separates the measured points. This enables visual assessment of the quality and origin of the jarkus measurements.

\section{Transects comparison view}

With the transects comparison view (Figure 3) it is possible to compare jarkus measurements in time for one location or in space for a specific year. This enables the user to look at profile development of a certain feature in time or space. Each measurement is now plotted by a single (unique) color. The tool offers various color scales to customize the image. 


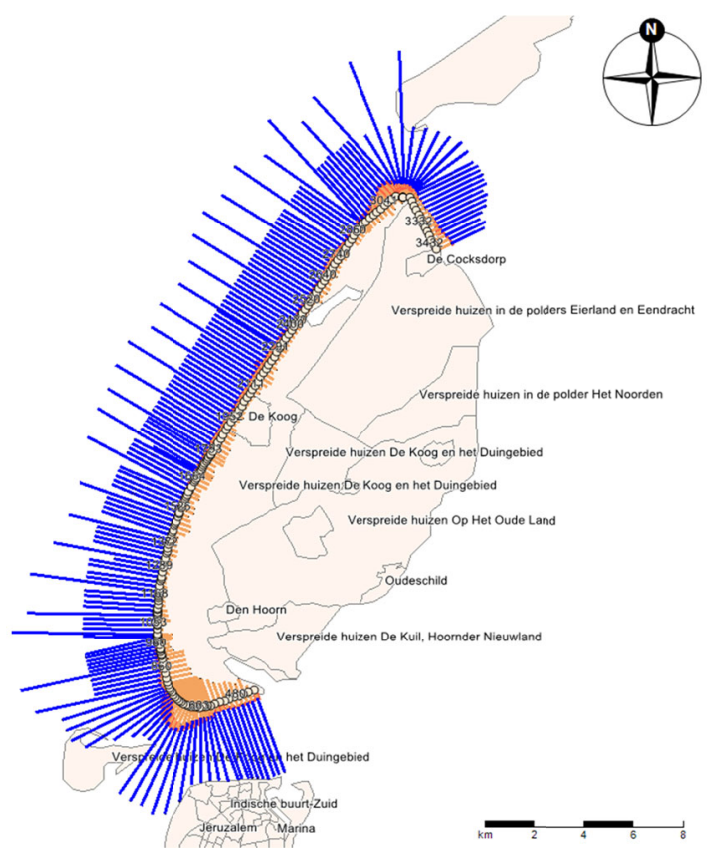

Figure 1. An example of a map in MorphAn showing an overview of the imported jarkus measurements for Texel. The map also contains a layer that shows the districts of Texel according to the Dutch cadastre (http://kadaster.nl/)

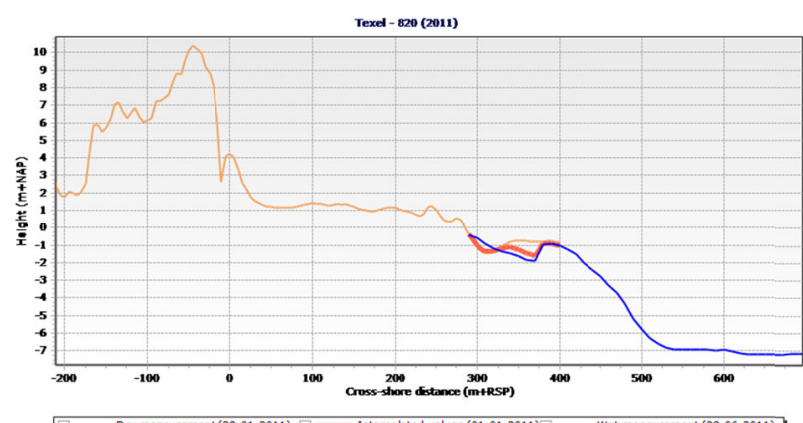

Figure 2. An example of a transect side view in MorphAn. The side view shows differences between measurements taken from land (sandy colored line), measurements taken from a vessel (blue line) and interpolated profile parts that are used to connect the wet and dry measurement when assessing the complete profile (tomato red line)

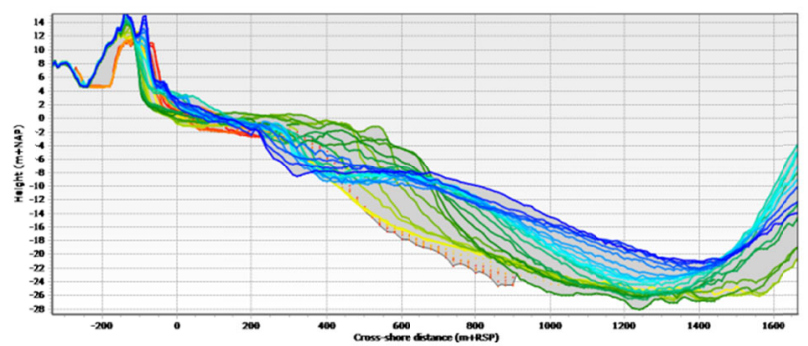

Figure 3. An example of a transect comparison plot in which measurements over a certain period on one location, or measurements for one year over multiple locations can be compared.

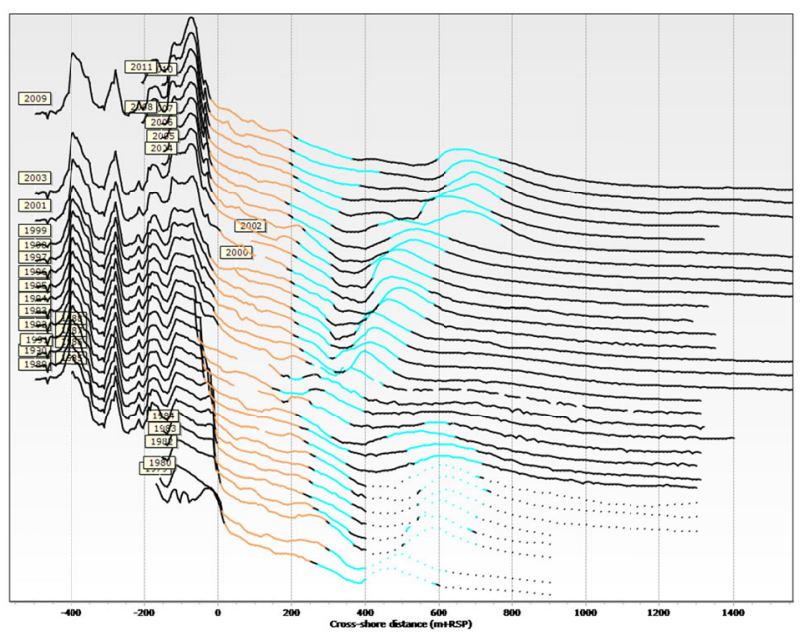

Figure 4. An example of a time stack representation of the beach development at one location. Brown and blue colors accentuate certain height regions of the profile. The blue part clearly shows a bar traveling offshore.

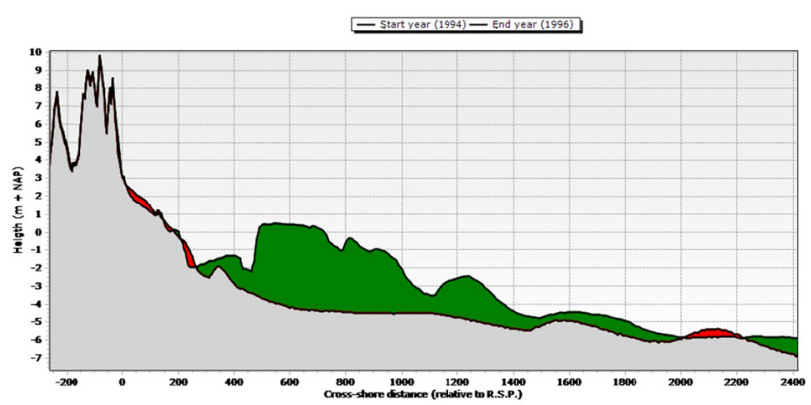

Figure 5. An example of a difference plot between two measurements. Green shows the parts of the profile that have accreted (or have been nourished) since the first measurement. The red parts indicate locations where the profile has eroded relative to the first measurement.

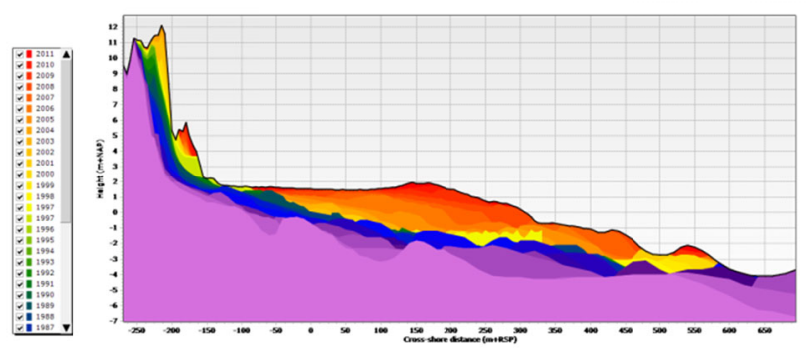

Figure 6. An example of a time history plot. The colored patches indicate the last year in which a change of the bathymetry was measured in that particular part of the profile. 


\section{Time stack view}

The time stack view is another way of looking at measurements in time at a certain location. In this plot each year is plotted a certain distance higher than the previous year. Due to the highlighting of specific heights in the profile, development of features in the profile becomes more visible. The time stack in Figure 4 for example shows the movement of breaker banks offshore.

\section{Time difference view}

Visualization of the morphological change between two years at a certain location can help the user understand the effect of large changes in the bathymetry. The time difference view (Figure 5) shows these differences as green (accretion) or red (erosive) areas. Due to its coupling with a time navigator development of these changes in time is quickly visualized. The example in Figure 6 shows a nourishment just below NAP. By holding the same reference year and changing the year of the compared jarkus measurement the development of the nourishment in time can be analyzed.

\section{Time history view}

Time history figures (Figure 6) show the development of the cross-shore profile at one location in time by plotting colored patches. The color represents the last year in which a change occurred in that particular part of the profile. With this tool the user gets an idea of the depth at which the profile actively changes through time.

\section{DUNE SAFETY ASSESSMENT}

Dutch dune safety assessment rules (TRDA2006) prescribe the use of Duros + for calculating dune erosion at the end of an extreme storm event. To assess dune safety, dune erosion due to this extreme event needs to be calculated for profile measurements over a period of fifteen years. To incorporate the yearly variation of the beach profile, the third worst result over these fifteen years is considered the normative erosion point.

MorphAn includes a model to calculate dune erosion with the Duros + model and visualize the results in order to inspect them (it implements Duros + ). Figure 7 shows a representation of such a calculation result for an individual measurement at Ameland. Next to the Duros+ model, MorphAn also implements the D++ model (Deltares, 2010).

To support determination of the normative erosion point, MorphAn also produces Rt-diagrams as described in the assessment rules (Figure 8). An Rt-diagram plots the calculated retreat points $(\mathrm{R})$ in time $(\mathrm{t})$ for a certain location and identifies the third worst point as the normative dune crest retreat point.

One of the added values of MorphAn with respect to existing tools is the ability to interactively display the calculation results on a map as well (Figure 9). This map representation uses the same feature as the previously discussed map overview. Combining the calculation results with other data therefore is relatively easy. Furthermore, selection of one ore more of the results on the map is synchronized with the representations of the calculated erosion and the Rt-diagram. This makes navigating through the results a fun job to do.

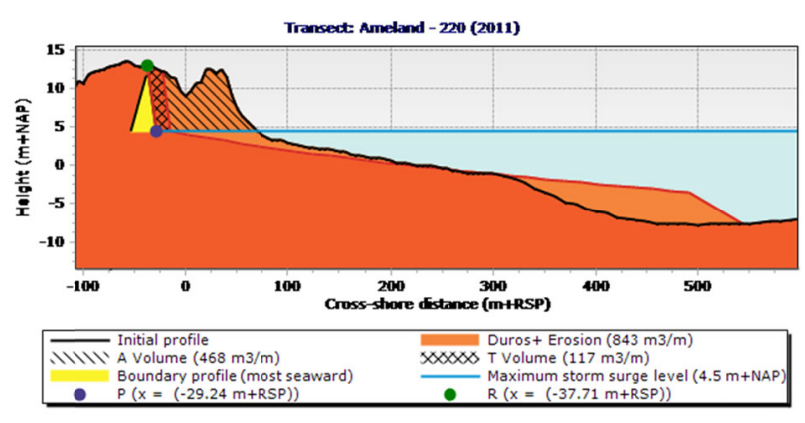

Figure 7. Representation of the result of a dune safety assessment calculation using Duros+ at Ameland as included in MorphAn. The figure also includes the calculated additional erosion and boundary profile.

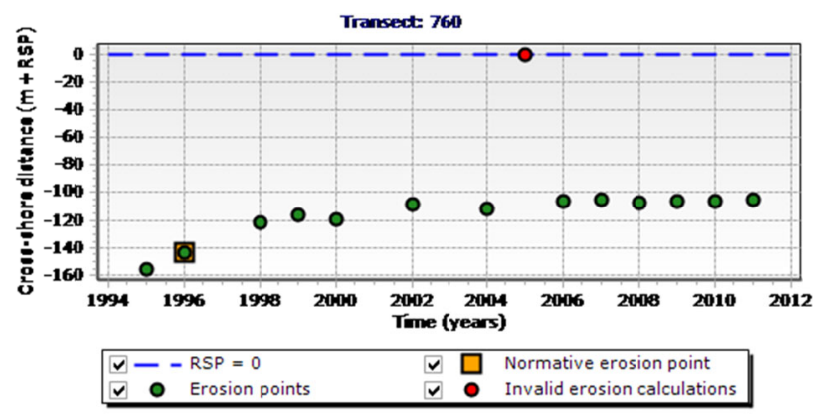

Figure 8. Visualization of the calculated retreat $(R)$ points in time. The normative retreat point is visualized as a squared symbol. MorphAn also distinguishes between questionable (orange), invalid (red) and valid (green) calculation results. This qualification is just a first pointer to the results that need some more examination before trusting the result.

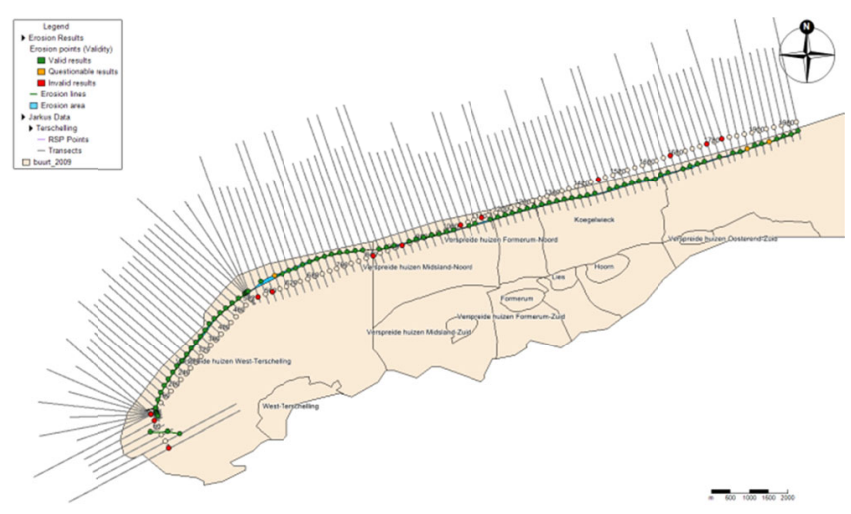

Figure 9. Map overview of the calculated retreat points. The map is coupled to a time navigator, which allows scrolling through time. The MorphAn overview also includes a layer that shows the normative retreat points. 


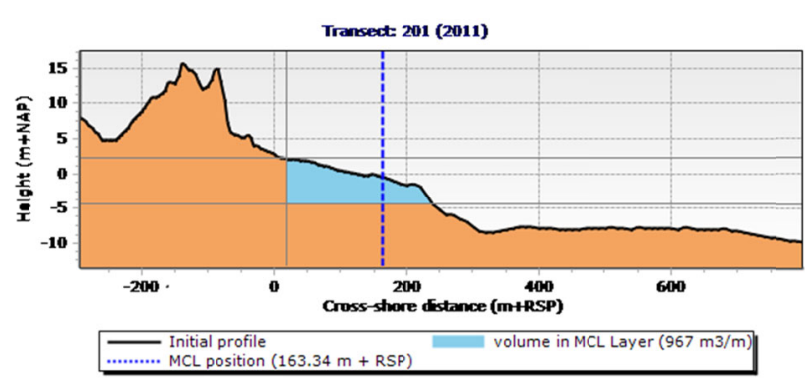

Figure 10. Representation of the calculated momentary coastline position (MKL) as included in MorphAn. The blue area visualizes the volume between the upper and lower boundary for determining the MKL position. The blue dashed line represents the MKL position.

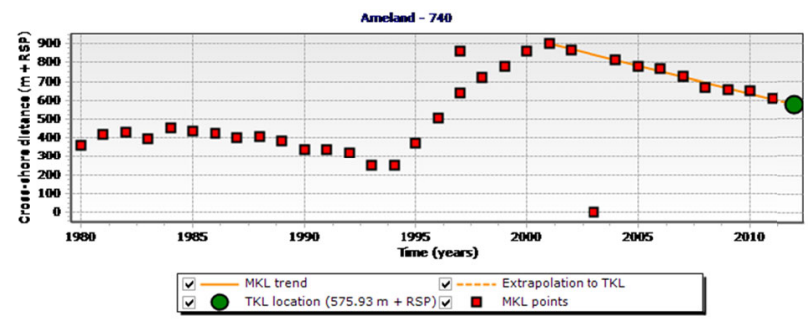

Figure 11. Representation of the MKL point in time for a location at Ameland. MorphAn also includes the calculated trend of the $M K L$ points by plotting an orange line. The green dot represents the predicted coastline TKL.

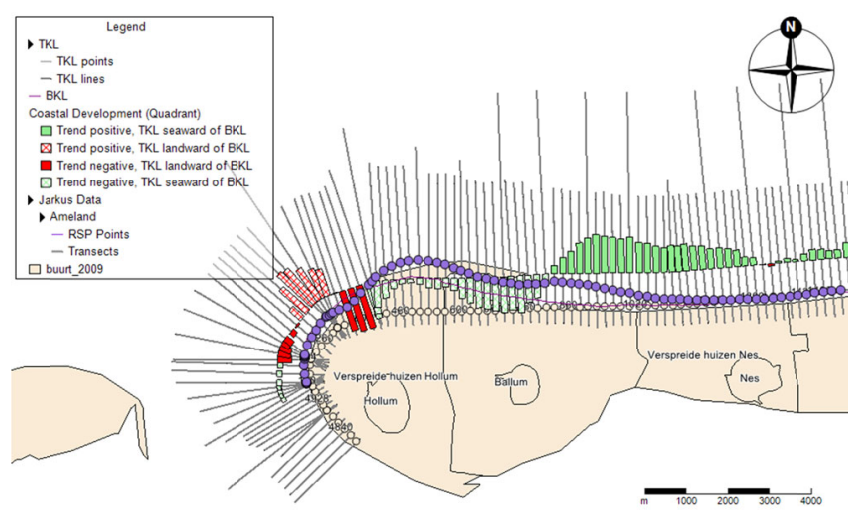

Figure 12. Map overview of the calculated MKL and TKL points. The map also includes a layer that expresses the trend of the $M K L$ relative to the basic coastline (BKL) by means of red and green bars. This layer looks similar to the presentation of $M K L$ trends in the kustlijnkaartenboek published by Rijkswaterstaat at www.kustlijnkaart.nl

\section{COASTAL DEVELOPMENT ASSESSMENT}

Next to dune safety assessment, MorphAn offers tools to assess the development of the coastline. A widely used indicator for the beach position is the momentary coastline position (MKL) defined as the volume of sediment between two elevation levels divided by the difference between these two levels. While assessing the development of the coastline these positions in time at a certain location are often used to calculate a trend and determine the expected coastline position (TKL) in a future year. Comparison with a reference coastline (BKL) ads to the decision whether or not there should be nourished at that particular location. Figures 10, 11 and 12 show the way MorphAn represents these calculation results. Figure 12 also contains a representation of the trend of the MKL position and its relation to the TKL similar to kustlijnkaarten produced by the Ministry of Infrastructure and the Environment (www.kustlijnkaart.nl). As the ministry initiated the development of MorphAn, it also plans on assessing the MKL development and determining the TKL with MorphAn in future.

\section{FUTURE DEVELOPMENT}

As most users will experience, MorphAn offers a lot of potential for further development of the supported operations. At this moment equipping MorphAn with the necessary tools to assess dune safety according to an expected new version of the assessment rules has a high priority. Next to that, attention is paid to expansion of the functionality to calculate beach development, like including a nourishment database and a module to automatically determine the best period for trend analysis. Although there is no direct coupling between MorphAn and the kustlijnkaarten produced by the ministry, in future calculation results can be written in an output format that can be used for creating these maps.

However, there are also possibilities for adding new features to MorphAn. One can think of including interfaces to other dune erosion models like XBeach (Roelvink et al., 2009) or DurosTA (Steetzel, 1993), but also expanding the capabilities of communication with other programs (export of results to Google Earth for example).

\section{CONCLUSIONS}

In this paper, we presented a new software tool, called "MorphAn" to facilitate analysis and assessment of safety and development of sandy coasts. The software is freely available and expected to become open source at the end of 2012. MorphAn supports data analysis in various ways as well as assessment of dune safety according to the Dutch assessment rules denoted in the TRDA2006 and assessment of the development of the momentary coastline. At this moment, MorphAn still offers lots of possibilities for expanding the functionality. We would love to hear all ideas about the possibilities to expand MorphAn in a way we did not think of yet.

\section{REFERENCES}

Deltares (2010), Ontwikkeling detailtoest duinen 2011 (D++), tussenrapportage SBW-Duinen. Deltares, 1202124-003).

Dano Roelvink, Ad Reniers, Ap van Dongeren, Jaap van Thiel de Vries, Robert McCall, Jamie Lescinski (2009), Modelling storm impacts on beaches, dunes and barrier islands, Coastal Engineering, 56(11-12),1133i-1152, ISSN:0378-3839, 10.1016/j.coastaleng.2009.08.006.

TRDA2006 (2007), Technisich rapport duinafslag, experisenetwerk waterveiligheid.

Henk Steetzel (1993). Cross-shore Transport during Storm Surges. 